\title{
Latest results on VBF and VBS processes from the CMS experiment
}

\section{Nadezda Chernyavskaya* on behalf of the CMS Collaboration}

Swiss Federal Institute of Technology in Zurich (ETHZ), Zurich, Switzerland

E-mail: nadezda.chernyavskaya@cern.ch

The latest measurements of the vector boson fusion (VBF) and vector boson scattering (VBS) processes from the CMS experiment are presented, using $36 \mathrm{fb}^{-1}$ data collected in proton-proton collisions at $\sqrt{s}=13 \mathrm{TeV}$ at the LHC. The measured total cross sections are compared to the theoretical predictions and limits are set on the presence of anomalous triple and quartic gauge couplings.

Sixth Annual Conference on Large Hadron Collider Physics (LHCP2018)

4-9 June 2018

Bologna, Italy

\footnotetext{
* Speaker.
} 


\section{Introduction}

Measurements of the pure electroweak (EWK) production of a vector boson in association with jets provide important tests of the EWK symmetry breaking (EWSB) mechanism in the standard model (SM). In the diboson vector boson scattering (VBS) processes the interference with the Higgs boson restores unitarity. Any deviation from the coupling between the Higgs boson and a vector boson would break this unitarity. Therefore, studying VBS processes allows to probe the role of the Higgs boson in such quartic couplings processes. The vector boson fusion (VBF) and VBS processes $\mathrm{V}(\mathrm{V})+\mathrm{jj}$ are very sensitive to physics beyond the $\mathrm{SM}(\mathrm{BSM})$ via anomalous triple and quartic gauge couplings (aTGC and aQGC), as well as production of the same final states through narrow resonances. In addition, the study of such EWK processes is an important benchmark to validate and cross check other VBF production modes such as the VBF production of the Higgs boson.

VBF and VBF processes have a very distinct signature. $\mathrm{V}(\mathrm{V})+\mathrm{jj}$ final states are characterized by a large dijet invariant mass and a large pseudorapidity separation between the tagging jets. The tagging jets originate from quarks as opposed to mostly gluon-originating QCD background jets. These features allow to better discriminate EWK signal from background. Another very distinctive property of the VBF process is that at leading order (LO) there is no QCD color exchanged in the pure EWK interaction, which leads to only very little additional radiation and hadronic activity outside of the color-connected region called the rapidity-gap. These features of low additional activity can be investigated in the $\mathrm{VBF} Z+\mathrm{jj}$ production as part of a more general investigation of the SM VBF processes that include the Higgs boson. Probing the jet activity in the signal pure region can shed some light on the modelling of the additional parton radiation from the tagging jets.

\section{VBF processes and triple gauge couplings}

The measurement of the EWK production of the $\mathrm{Z}$ boson in association with two jets was performed by the CMS experiment [1] at $\sqrt{s}=13 \mathrm{TeV}$ with the integrated luminosity $36 \mathrm{fb}^{-1}$ [2]. The EWK signal is defined in the kinematic region with dilepton mass $M_{l l}>50 \mathrm{GeV}$, parton transverse momentum $p_{T}(j)>25 \mathrm{GeV}$, and diparton mass $M_{j j}>120 \mathrm{GeV}$, where the cross section of the $l l \mathrm{jj}$ final state (with $l=e$ or $\mu$ ) is expected to be $\sigma_{L O}($ EWK lljj) $=543 \pm 7$ (scale) \pm 22 (PDF) fb. The main background is Drell-Yan (DY) plus jets events, DY Zjj. Events are required to have at least two jets with $p_{T}$ requirements on the leading and subleading jets $p_{T}\left(j_{1}\right)>50 \mathrm{GeV}, p_{T}\left(j_{2}\right)>30$ $\mathrm{GeV},|\eta|<4.7$, and dijet invariant mass $M_{j j}>200 \mathrm{GeV}$. In addition, events are required to have at least two leptons (electrons or muons) with invariant mass compatible with the $\mathrm{Z}$ boson mass within $15 \mathrm{GeV}$. The leading lepton and subleading leptons are required to have $|\eta|<2.4$ and $p_{T}>$ $30 \mathrm{GeV}, p_{T}>20 \mathrm{GeV}$ for leading and subleading leptons respectively. To enhance the signal and separate it from the background, a boosted decision tree (BDT) is trained on the most discriminating features of the VBF production. Figure 1 (left) shows the distribution of the transformed BDT output for the dimuon channel. Good agreement with data is observed.

A binned maximum likelihood fit is performed as a function of the BDT output. Nuisance parameters are used to constrain the background normalization and account for other systematic 
uncertainties. Dominant systematic uncertainties are the jet energy scale uncertainty and the one associated to the simulated sample size on the experimental side, and the background QCD scale uncertainties on the theoretical side. The results obtained are compatible among the dilepton channels and the measured cross sections are the following: $\sigma(\mathrm{EWK} \mu \mu \mathrm{jj})=524 \pm 23($ stat $) \pm 61$ (syst) $\mathrm{fb} ; \sigma($ EWK eejj $)=521 \pm 34($ stat $) \pm 68($ syst $) \mathrm{fb} ; \sigma($ EWK lijj $)=534 \pm 20($ stat $) \pm 57($ syst $) \mathrm{fb}$, in agreement with the SM prediction.

Having established the EWK signal, the properties of the additional hadronic activity in the rapidity gap can be studied. A substantial suppression of additional radiation is expected in signal events in contrast to QCD-induced production of jets in the main DY Zjj background. A signalenriched sample is selected by applying a cut BDT $>0.92$, which yields approximately the same number of signal and background events. Additional jet activity is studied using jets with $p_{T}>15$ $\mathrm{GeV}$, while for additional charged hadronic activity a collection of high-purity tracks [5] with $p_{T}>$ $0.3 \mathrm{GeV}$ associated with the main primary vertex in the event is formed. These tracks are clustered in "soft track-jets" using the anti- $\mathrm{k}_{T}$ clustering algorithm [6] with distance parameter of $\mathrm{R}=0.4$. Using the "soft track-jets" allows to reconstruct energy to as low as a few GeV. Good agreement is observed between data and and the simulation for additional jet and hadronic activity.
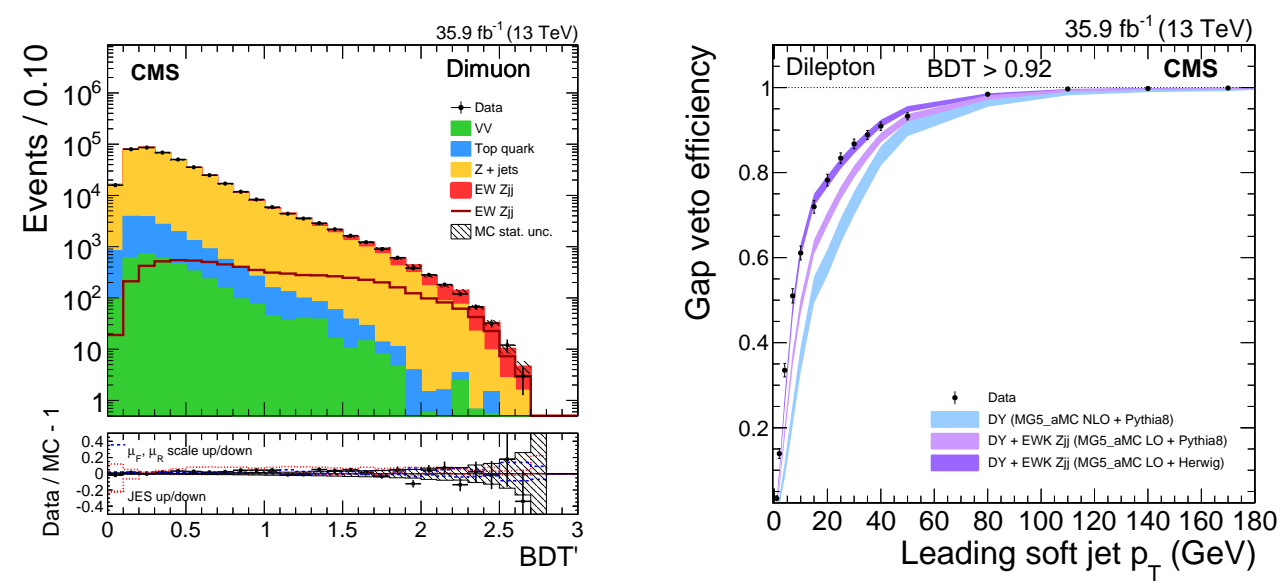

Figure 1: (left) Distributions for the BDT discriminants in dimuon events. The contributions from the different background sources and the signal are shown stacked, with data points superimposed. The lower panels show the relative difference between the data and expectations. (right) Efficiency of a gap activity veto in dielectron and dimuon events with BDT $>0.92$, as a function of the leading soft jet $p_{T}$. Data points are compared to Monte Carlo (MC) expectations with only DY events, including for signal either the PYTHIA or the HERWIG ++ parton shower models. The bands represent the MC statistical uncertainty [2].

Measurements of veto efficiency can quantify how reliable the modelling is in a signal-enriched region. The efficiency of a gap activity veto corresponds to the fraction of events which do not have reconstructed kinematic quantities above a given threshold. The veto efficiencies are studied as a function of the applied threshold of the transverse momentum $p_{T}$ and the scalar $p_{T}$ sum of jets $H_{T}$ and 'soft track-jets'. The data are compared to two parton shower predictions PYTHIA and HERWIG ++ [4], and found to be in agreement with both models. In Figure 1 (right) the veto efficiency of combined dilepton events is shown as a function of the $p_{T}$ of the leading soft jet threshold. The 
data seem to prefer the signal model with HERWIG ++ parton showering at low gap activity values while for larger gap activities the PYTHIA [3] description might be better.

Anomalous triple gauge couplings are studied in the the framework of an effective field theory (EFT). The VBF Z + jj production mode has high sensitivity to the $c_{W W W} / \Lambda^{2}$ and $c_{W} / \Lambda^{2}$ coupling parameters. A simultaneous binned likelihood fit is performed as a function of the transverse momentum of the $\mathrm{Z}$ boson $p_{T Z}$ to extract the limits on the aTGCs. No significant deviation from the SM is observed and the limits on the EFT parameters are presented in Table 1, including the most stringent constraints on $c_{W W W} / \Lambda^{2}$ parameter to date.

Table 1: One-dimensional limits on the aTGC EFT parameters at 95\% CL.

\begin{tabular}{c|c|c} 
Coupling parameter & Expected 95\% CL interval $\left(\mathrm{TeV}^{-2}\right)$ & Observed $95 \% \mathrm{CL}$ interval $\left(\mathrm{TeV}^{-2}\right)$ \\
\hline$c_{W W W} / \Lambda^{2}$ & {$[-3.7,3.6]$} & {$[-2.6,2.6]$} \\
$c_{W} / \Lambda^{2}$ & {$[-12.6,14.7]$} & {$[-8.4,10.1]$}
\end{tabular}

\section{VBS processes and quartic gauge couplings}

The VBS processes have lower cross sections than the VBF processes and thus had not been observed at a discovery level until recently. Observation of EWK production of same-sign W boson pairs in the two jet and two same-sign lepton final state at $\sqrt{s}=13 \mathrm{TeV}$ was performed by the CMS [7]. This same-sign $\mathrm{W}$ boson pair has the largest cross section and the cleanest signature among the diboson production processes. The main background contribution is QCD-induced same-sign W boson pairs, top quark, Drell-Yan, and WZ background contributions.

The VBS topology is enhanced by requiring at least two jets with $p_{T}(j)>30 \mathrm{GeV},|\eta|<5.0$, dijet invariant mass $M_{j j}>500 \mathrm{GeV}$, pseudorapidity separation $\left|\Delta \eta_{j j}\right|>2.5$ and $\max \left(z_{l}^{*}\right)<0.75$ where $z_{l}^{*}=\left|\eta_{l}-\left(\eta_{j 1}+\eta_{j 2}\right) / 2\right| /\left|\Delta \eta_{j j}\right|$ is the Zeppenfeld variable [8]. Exactly 2 leptons (muons or electrons) should be present and are required to have $p_{T}>25 \mathrm{GeV}, p_{T}>20 \mathrm{GeV}$ for the leading and subleading leptons respectively.

A control region is defined to estimate the WZ background contribution. A $W Z \rightarrow 3 l v$ control region is defined by requiring an additional lepton with $p_{T}>10 \mathrm{GeV}$ and an opposite-sign same-flavour lepton pair with an invariant mass consistent with the $\mathrm{Z}$ boson mass.

The signal contribution is extracted from a two-dimensional fit of the $M_{j j}$ and $M_{l l}$ distributions. The fit is performed simultaneously in the signal and WZ control regions. Only $M_{j j}$ is used in the fit of the WZ control region. The observed (expected) statistical significance of the signal is 5.5 (5.7) standard deviations. The ratio of the measured signal event yield to that expected from the $\mathrm{SM}$ is $\mu=0.90 \pm 0.20$. The measured fiducial cross section is found to be $\sigma_{\text {fid }}\left(\mathrm{W}^{ \pm} \mathrm{Wjj}\right)=$ $3.83 \pm 0.66$ (stat) \pm 0.35 (syst) fb and is in agreement with the SM prediction.

Apart from the cross section measurement, anomalous QGC can be studies by performing a likelihood analysis of $M_{l l}$ distributions in signal and control regions. No anomalous signal is found and stringent limits are set. In addition to the limits on aQGCS, a doubly-charged Higgs resonance search is performed in the same-sign WW final state with the VBF topology. The GeorgiMachacek model of Higgs triplets [9] is considered. A simultaneous two-dimensional fit to $M_{j j}$ 
and $M_{l l}$ distributions in signal and control regions is performed. No BSM signal is observed and the limits on the double-charged Higgs production and decay are set. Figure 2 shows the $M_{j j}$ distribution and the limits on doubly charged Higgs production.
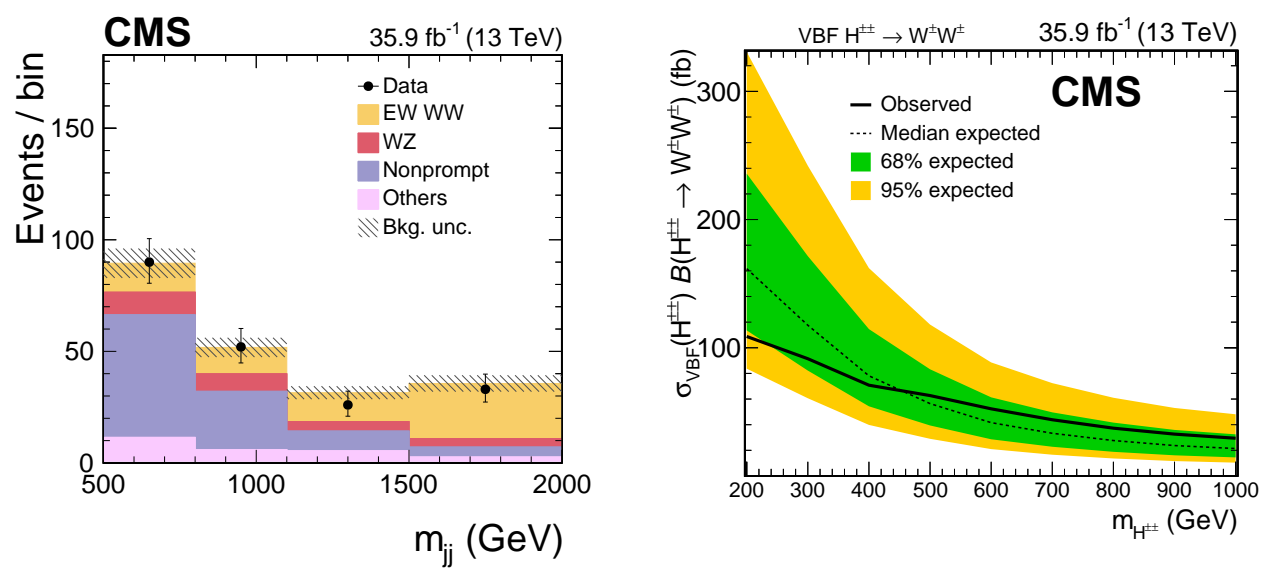

Figure 2: (left) The $M_{j j}$ distribution of same-sign WW $+\mathrm{jj}$ final states. The contributions from the different background sources and the signal are shown stacked, with data points superimposed. (right) Expected and observed 95\% C.L. upper limits on the cross section times branching fraction of the doubly charged Higgs boson as a function of the doubly charged Higgs boson mass.

Another rare diboson VBS process, $\mathrm{ZZ}+\mathrm{jj}$ in the four-lepton final state, has been measured for the first time by the CMS [10]. This result was finally feasible with the $36 \mathrm{fb}^{-1}$ data collected at $\sqrt{s}=13 \mathrm{TeV}$ at LHC. Despite a low cross section and a low $Z \rightarrow l l$ branching fraction, due to a clean leptonic final state and a precise knowledge of the scattering energy, this channel provides a favourable topology to study EWSB. The dominant background is QCD-induced production of two $\mathrm{Z}$ bosons in association with jets.

Events are required to have four leptons with invariant mass consistent with the mass of the $\mathrm{Z}$ boson within $30 \mathrm{GeV}$ mass. At least two jets are required with $p_{T}>30 \mathrm{GeV}$ and invariant mass $M_{j j}>100$ $\mathrm{GeV}$. To enhance the separation between signal and background a BDT trained on distinctive VBS features is used. The QCD background modelling is verified in the QCD-enriched control region, that is defined by selecting events with the anti-VBF topology cuts $M_{j j}<400 \mathrm{GeV}$ and $\left|\Delta \eta_{j j}\right|<$ 2.4. Good agreement between data and SM expectation is observed.

The signal contribution is extracted via a maximum-likelihood fit to the BDT distribution, that is shown in Figure 3 (left). The QCD normalization is constrained by the background-enriched region of the BDT distribution. The measured fiducial cross section is in agreement with the SM expectation $\sigma_{\text {fid }}(\mathrm{ZZjj})=0.40_{-0.16}^{+0.21}(\text { stat })_{-0.09}^{+0.13}($ syst $) \mathrm{fb}$. The observed (expected) significance is 2.7 (1.6) standard deviations.

The events in the $\mathrm{ZZ}+\mathrm{jj}$ selection are used to constrain aQGCs in the EFT approach. The ZZ $+\mathrm{jj}$ channel is sensitive to the operators T0, T1, T2, and neutral current operators T8 and T9 [11]. The $M_{Z Z}$ distribution is used to constrain aQGC parameters in a likelihood fit. Figure 3 (right) 
shows the expected $M_{Z Z}$ distribution for the SM and two aQGC scenarios. No anomalous signal is observed and the most stringent limits to date on the aQGC parameters $f_{T 0,1,2} / \Lambda^{4}$ and $f_{T 8,9} / \Lambda^{4}$ are derived.
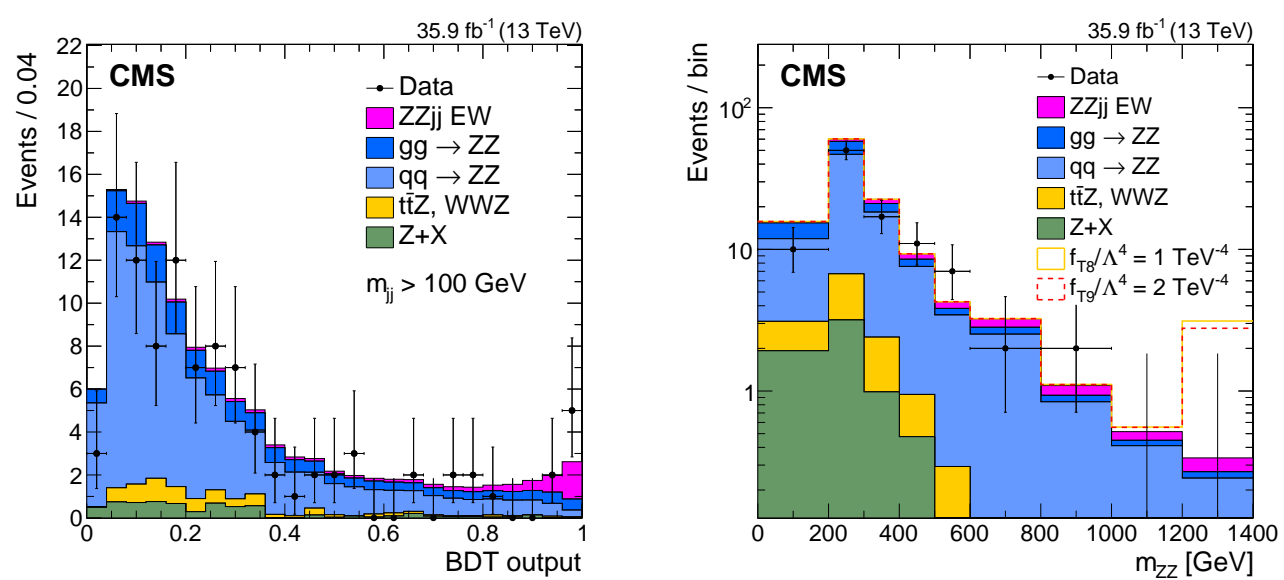

Figure 3: (left) Distribution of the BDT discriminant for the selected $\mathrm{ZZ}+\mathrm{jj}$ events. (right) The $M_{\mathrm{ZZ}}$ distribution for the selected $\mathrm{ZZ}+\mathrm{jj}$ events together with the SM prediction and two hypotheses for the aQGC coupling strengths.

\section{Conclusions}

The latest results on VBS and VBF measurements at $\sqrt{s}=13 \mathrm{TeV}$ from the CMS experiment do not show any deviation from SM predictions, but strong limits on the aTGCs and aQGCs are set. With $\sqrt{s}=13 \mathrm{TeV}$ data at the LHC the VBS processes are becoming accessible. The first observation of the diboson VBF process in the same-sign $\mathrm{W}$ bosons final state and the first measurement of the $\mathrm{ZZ}+\mathrm{jj}$ process are presented. The $\mathrm{VBF} \mathrm{Z}+\mathrm{jj}$ process is measured with $10 \%$ precision and the measured cross section is in agreement with the SM. Studies of the VBF processes are able to validate the modelling of rapidity gap activity observables in a high-purity VBF topology.

\section{References}

[1] S. Chatrchyan et al. [CMS Collaboration], JINST 3 (2008) S08004. doi:10.1088/1748-0221/3/08/S08004

[2] A. M. Sirunyan et al. [CMS Collaboration], Eur. Phys. J. C 78 (2018), 589 doi:10.1140/epjc/s10052-018-6049-9 [arXiv:1712.09814 [hep-ex]].

[3] T. Sjöstrand, S. Mrenna and P. Z. Skands, JHEP 0605 (2006) 026 doi:10.1088/1126-6708/2006/05/026 [hep-ph/0603175].

[4] M. Bahr et al., Eur. Phys. J. C 58 (2008) 639 doi:10.1140/epjc/s10052-008-0798-9 [arXiv:0803.0883 [hep-ph]].

[5] CMS Collaboration, CMS-PAS-TRK-10-005, http://cds.cern.ch/record/1279383. 
[6] M. Cacciari, G. P. Salam and G. Soyez, JHEP 0804 (2008) 063 doi:10.1088/1126-6708/2008/04/063 [arXiv:0802.1189 [hep-ph]].

[7] A. M. Sirunyan et al. [CMS Collaboration], Phys. Rev. Lett. 120 (2018), 081801 doi:10.1103/PhysRevLett.120.081801 [arXiv:1709.05822 [hep-ex]].

[8] D. L. Rainwater, R. Szalapski and D. Zeppenfeld, Phys. Rev. D 54 (1996) 6680 doi:10.1103/PhysRevD.54.6680 [hep-ph/9605444].

[9] H. Georgi and M. Machacek, Nucl. Phys. B 262 (1985) 463. doi:10.1016/0550-3213(85)90325-6

[10] A. M. Sirunyan et al. [CMS Collaboration], Phys. Lett. B 774 (2017) 682 doi:10.1016/j.physletb.2017.10.020 [arXiv:1708.02812 [hep-ex]].

[11] O. J. P. Eboli, M. C. Gonzalez-Garcia and J. K. Mizukoshi, Phys. Rev. D 74 (2006) 073005 doi:10.1103/PhysRevD.74.073005 [hep-ph/0606118]. 